\title{
Critical Analysis of Contribution of Emotional Literacy on Relationships
}

\author{
Archana Shirpurkar \\ Course Coordinator, Clinical Research, R. Jhunjhunwala College, Ghatkopar West, Mumbai. \\ Corresponding author: Archana Shirpurkar \\ Email - naad_archana@yahoo.co.in
}

\begin{abstract}
Background: The aim of this paper is to emphasize the role of emotional literacy in various facets of relationships. Our mental health depends on understanding various emotional dimensions in our life. In this study, factors influencing emotional literacy are analyzed to improve interpersonal relationships and academic success as well.

Methods: The research study suggests a model for management of emotions to improve emotional literacy. In this research study, based on previous research and formal and informal interview settings, an attempt has been made to develop a generalized approach to enhance emotional literacy. Questionnaires were designed to collect qualitative data. Participants were divided into control group and experimental group. Qualitative analysis is interpretative as responses were implicit and could be understood through deep and repeated familiarity. The model is designed to manage eight basic emotions. The model is based on identifying, registering, reasoning, articulating, managing and responding to emotions.

Results: This study has aimed to develop an approach to enhance emotional literacy. In this approach, the focus is on individual's emotions. The effective use of this approach would help for individual's mental health. Emotional intelligence could be further developed by understanding others' emotions and dealing with them.

Conclusion:Model of Emotional literacy could be used as a tool for improving interpersonal and social relationships, academic success and overall well-being.
\end{abstract}

Keywords: Mental health, Emotions, Relationship, Emotional literacy.

(Paper received $-2^{\text {nd }}$ January 2018, Peer review completed $-6^{\text {th }}$ January 2018)

(Accepted $-10^{\text {th }}$ January 2018)

\section{INTRODUCTION}

Human growth and development can be explained in different ways according to biological growth, cognitive growth, emotional growth, spiritual growth etc. The concept of emotional development has been an area of interest for researchers over several years. Globalization, modernization, cultural changes, social changes are some of the factors influencing and challenging understanding of emotional development. Many theories are put forth by psychoanalysts which include Freud's psychodynamic theory, Bandura's social learning theory, Erikson's psychological theory etc. Relating these theories in practical, in this ever-changing world is a challenge for psychologists and psychoanalysts. To articulate emotional development in the current scenario, it is necessary to have deeper understanding of emotional learning or literacy.

The purpose of this study is to unearth new productive approach in emotional learning process. The study aims to articulate stages of emotional learning, emotional competence skills, factors affecting these skills 
and an approach to enhance emotional literacy. The term 'Emotional Literacy' is the ability to understand and express feelings. Emotional learning is a continuous process which involves identifying, registering, reasoning, articulating, managing and responding to the situation. Emotions may be more complicated to understand as it depends on various factors like attitude, individual perception, situation, relationships, and environment. Emotional literacy could be a tool for better inter-personal relationships, sociopsychological development, cognitive development, creativity and happiness. Understanding and enhancing emotional literacy improves confidence, efficiency and productivity. Previous studies have shown that lack of emotional learning may have negative impact like learning and behavioural disorders [1].

\section{Theoretical framework}

'Emotion' is an umbrella term, defined by various researchers. Psychologists consider 'emotion' as a complex psychological event that involves feelings comprising physiological and behavioural responses. 'James-Lange theory' describes the origin and nature of emotions put forth by William James and Carl Lange in the late $19^{\text {th }}$ century. This followed by several theories like Cannon- Bard Theory, Schachter Singer theory etc. 'Appraisal theory' was the first contemporary theory developed by an American psychologist Magda Arnold. Emotions are classified by researchers in different ways. Ortony and Turner compiled basic emotions identified by various researchers. Further, Shaver, Kirson and O'Connor further developed deeper list of emotions [1]. Robert Plutchik developed 'Theory of emotion' in 1980 explained in ten postulates. His psycho-evolutionary theory is one of the most influential classification approach for emotional responses [2].

This research study is focused on emotional learning process and attempts to develop an approach to 'emotional literacy'. Here, it is necessary to define 'Emotional literacy' and 'Emotional intelligence'. Emotional literacy is the capacity to register and acknowledge emotional responses to the particular situation and to recognize the way they influence individual's thoughts and actions. In simple words, emotional literacy is learning the language of feelings and emotions. Emotional intelligence on the other hand is a person's ability to empathize with other's emotions and effective management of emotions. This could be considered as 'best practices' in effective management of emotions. This includes acknowledgement of individual's and others' emotions.

\section{Types of emotions}

'Emotion' is energy in motion. Emotions are generally triggered by an event, person or thoughts and are expressed. This causes a positive or negative impact on an individual or others. Over a period, pattern of expression of emotions develops 'behaviour'. Based on favourable or unfavourable situation, positive or negative emotions are experienced and create an impact.

\begin{tabular}{|l|l|l|}
\hline Situation & Basic emotion & Impact \\
\hline Favourable & 1. joy & celebrate \\
\hline & 2. trust & connect with the person \\
\hline & 3.surprise & focus on new thing \\
\hline & 4.anticipation & look forward \\
\hline Unfavorable & 5. anger & fight against the problem \\
\hline & 6. fear & protect ourselves from danger \\
\hline & 7. sadness & look for support \\
\hline & 8. disgust & reject unwanted situations \\
\hline
\end{tabular}

Table 1 - Relation between situation, basic emotion and impact

Concept of emotions has always been an area of interest and research as it is closely related to mental health. Plutchik's wheel of emotions illustrating eight basic emotions is used as a tool for this study for better understanding and enhancing emotional literacy. Plutchik proposed eight basic emotions, anger, 
fear, sadness, disgust, surprise, anticipation, trust and joy which are illustrated in a wheel model to show their relatedness. Joy vs. sadness, anger vs. fear, trust vs. disgust and surprise vs. anticipation shows connections between the idea of emotion circle and colour wheel. The extension of basic emotions is given as follows -

1. Anger - annoyance - rage

2. Fear - apprehension - terror

3. Sadness - pensiveness -grief

4. Disgust - boredom - loathing

5. Surprise - distraction - amazement

6. Anticipation -interest - vigilance

7. Trust - acceptance-admiration

8. Joy - serenity - ecstasy

\section{Impact of emotions}

Emotions are broadly categorized as positive (joy, happiness, trust) and negative (sadness, anger, fear) emotions. Sometimes individual may experience combination or overlap of both. Though positive and negative emotions seem to be incompatible, a balance of both is necessary for mental health.

Some of the good effects of positive emotions could be improving thoughts and actions, enhancing resilience, minimizing negative thoughts, improves problem-solving and creativity and so on. These emotions should be cultivated and channelized. However, more intense and persistent positive emotions may create over-anxiety.

Negative emotions are completely natural. Negative emotions, if not managed properly, could lead to repression. Negative emotions may cause stress, affect mental and physical health, affect behaviour and relationships adversely, affect decision making and problem-solving capacity, substance abuse etc. If negative emotions are identified and contemplated in right context, they could be used positively. Negative emotions may help to think deeply, can facilitate learning and knowledge about the world, help in coping with the situation and increase preparedness.

\section{Stages of emotional development}

The stages of emotional development considered for this study are generalized as infancy, childhood, adolescence and adulthood. Emotional development milestones developed by Psychologist, Erick Erickson for each stage are given below [3]:

1. Infancy - Infancy stage is explained as the most fundamental stage exhibiting 'trust vs. mistrust'. Child's emotion starts developing from birth. Infants start showing 'social smile' around age 2 to 3 months and spontaneous laugh around 4 months. By the age of one year, babies show wide variety of emotions like anxiety, sadness, anger and distress.

2. Childhood - Childhood has 3 phases. Early childhood (1 to 3 years) is the concept of 'autonomy vs. shame' which is the beginning of child's independence. Pre-school phase (3 to 6 years) is about 'initiative vs. guilt' which emphasizes sense of achievement. School age (6 to 12 years) develops a sense of 'industry vs. inferiority' which is about comparison with peers.

3. Adolescence - (13 to 19 years) 'Identity vs. role confusion' is predominant during adolescence stage. Questioning self, exploring self-identity and confusion about the ideas and concepts are main characteristics of this stage. This stage is also termed as 'identity crisis'. This is due to the radical change during puberty. If rightly addressed, individual can freely experiment and explore the meaning of identity.

4. Adulthood - Adulthood begins at the age of 20. In early and middle adulthood, physical and emotional changes take place due to primary and secondary aging.

a) Early adulthood - (ages 20 to 39) This phase, termed as 'intimacy and isolation' is most crucial for enhancing emotional literacy. During this period, cognition and emotional development stabilize. Individuals start defining their ideas and concepts depending on their experiences, situation, culture and environment. Emotions depend on sense of productivity and achievement. The process of emotional development may be crystallized or fluid and 
depends on gathered knowledge and experiences. People face the crisis of being alone versus being involved in meaningful relationships. During this period, socio- emotional factors in work and family life play an important role.

b) Middle adulthood - (ages 40 to 64) 'Generativity vs. stagnation' phase, which revolves around a person's sense of purpose and sense of contribution to the world. Factors contributing to emotional management are activities at workplace, inter-personal relationships, social connectedness and culture.

c) Late adulthood - (ages 65 and onward) During this period, socio- emotional factors in work and family life play an important role. Emotions depend on sense of productivity and achievement. Erikson has coined this stage as 'ego integrity vs. despair'. Ego integrity is acceptance of victories as well as defeats.

\begin{tabular}{|c|c|c|}
\hline Stage of life & Influencers & Approach \\
\hline Infants & 1. Parents & $\begin{array}{l}\text { 1. Warm, positive parenting } \\
\text { 2. Talking, singing, cuddling, playing } \\
\text { 3. Immediate attention to infant's needs } \\
\text { 4. Avoid separation anxiety }\end{array}$ \\
\hline Children & $\begin{array}{ll}\text { 1. } & \text { Self } \\
\text { 2. } & \text { Parents } \\
\text { 3. } & \text { Teachers } \\
\text { 4. } & \text { Relatives } \\
\text { 5. } & \text { Peer }\end{array}$ & $\begin{array}{l}\text { 1. Optimism } \\
\text { 2. Support and encouragement } \\
\text { 3. Unconditional love and respect } \\
\text { 4. Avoid comparison } \\
\text { 5. Concept of independence }\end{array}$ \\
\hline Adolescents & $\begin{array}{ll}\text { 1. } & \text { Self } \\
\text { 2. } & \text { Parents } \\
\text { 3. } & \text { Teachers } \\
\text { 4. } & \text { Relatives } \\
\text { 5. } & \text { Peer }\end{array}$ & $\begin{array}{l}\text { 1. Optimism } \\
\text { 2. Support and encouragement } \\
\text { 3. Unconditional love and respect } \\
\text { 4. Avoid comparison } \\
\text { 5. Concept of self, emotions }\end{array}$ \\
\hline Adults & $\begin{array}{ll}\text { 1. } & \text { Parents } \\
\text { 2. } & \text { Teachers } \\
\text { 3. } & \text { Relatives } \\
\text { 4. } & \text { Peer } \\
\text { 5. } & \text { Colleagues }\end{array}$ & $\begin{array}{l}\text { 1. Optimism } \\
\text { 2. Support and encouragement } \\
\text { 3. Unconditional love and respect } \\
\text { 4. Avoid comparison } \\
\text { 5. Management of emotions }\end{array}$ \\
\hline
\end{tabular}

Table 2 - Stages of emotional development

\section{Effects of emotional illiteracy or disturbance}

Emotions can adversely affect mental, physical and cognitive growth, if not taken care of or ignored for a longer time. Prevention of emotional disturbance is possible by identification and intervention at an early age. Parents, specially mothers play a key role in developing emotional literacy in infants. During childhood and adolescence, parents as well as teachers can significantly help in emotional literacy. Hormonal and social changes add up to emotional disturbance during adolescence. Undiagnosed and untreated issues may lead to hyperactivity, aggression, withdrawal, immaturity, learning difficulties, distorted thinking and excessive anxiety. In adults, level of maturity and self-learning becomes necessary in emotional management. If ignored in childhood, emotional disorders surface as major concerns in adults which include -

1. Inability to maintain healthy interpersonal relationships

2. Inability to cope with adverse situations 
3. Inappropriate responses to normal situations

4. Aggressive attitude

5. Slows down cognitive development

6. Increase in negative emotions beyond control

7. Physical illness

8. Unhappiness or depression

\section{An approach to enhance emotional literacy}

Emotions are the feelings that happen to an individual which is associated with a situation or a person [4]. Emotions could be biologically pre-determined, situational, temporary, permanent, simple or complex. Experience of emotions is a step-wise process. Initially, an individual notice that something happened. This is an emotion in terms of information and energy. Emotion in terms of message is carried to the brain, brain decodes and sends back the response. An individual reacts to the situation which shows an impact. If the response becomes a pattern for similar situations, it becomes a behaviour $[5,6]$.

\section{METHODOLOGY}

Typically, Research was conducted on children and adolescents. Environment is carefully designed to closely monitor situations. As a tutor, children and adolescents were assessed for behavior, aptitude, emotional quotient. Children of age group eight to twelve and adolescents of age thirteen to sixteen were selected and randomly assigned to experimental or control group. Parents were interviewed to assess the culture, family background and environment at home. Control group participants were tutored in a normal routine. Experimental participants were tutored with intervention. Initially emotions and reactions to situations were analyzed for both the groups for particular situations. Experimental group was counselled for managing different types of emotions in a play-way method. Questionnaires were designed to collect qualitative data. The data was interpreted to draw a conclusion. In this research study, based on previous research and formal and informal interview settings, an attempt has been made to develop a generalized approach to enhance emotional literacy. Qualitative analysis is interpretative as responses were implicit and could be understood through deep and repeated familiarity. The approach designed was used for experimental group. Each step in the approach is carefully and repeatedly carried out identifying problems associated with each participant. At the end of the study, observations show interesting and positive results. The approach was found to be beneficial more for adolescents. For children below age of 12, showed good response after repeated attempts.

This approach could be adapted specifically for children, adolescence and adults. Influencers like parents, teachers, mentors or counsellors could use this approach effectively. The approach includes following six steps.

1. Identify - Individual faces a situation and experiences feelings. Perception of the situation depends on thinking process and previous experiences. As a first step, it is essential to identify that something is happening as expected or different and feelings are surfacing.

2. Register - Paying attention and acknowledgement of the situation should be the next step. Decoding the situation and basic emotions will help to find the meaning behind the feelings. Analyze intensity and complexity (combination of variety of emotions - mixed feelings) of the emotions. Do not ignore any emotion.

3. Reasoning - Curiosity to find out the reason behind the situation will lead to questioning about the situation and associated emotions. Reasoning will give answer to the question, 'if situation or emotions could have been controlled'. Considering previous response to the similar situation would help in reasoning.

4. Articulate - This step involves analyzing emotions with pragmatic thinking process. Confusion about emotions, overlap of emotions need to be segregated. It is necessary to prioritize and evaluate importance of situation and emotions. This will help to deduce anticipated consequences.

5. Manage - After deliberations, individual need to take control of emotions. This could be a challenge or a threat. Find out options to deal with basic emotions, choose best solution. 
Individual should take entire responsibility of the choice. In case of complicated situation and mixed emotions, consult influencers like parents, teachers or counsellors.

6. Respond - It is entirely individual's choice whether to respond or not. Individual can choose to express emotions in subtle way, act before the situation aggravates. It is essential to respond accordingly with suitable gestures.

\section{Emotions}

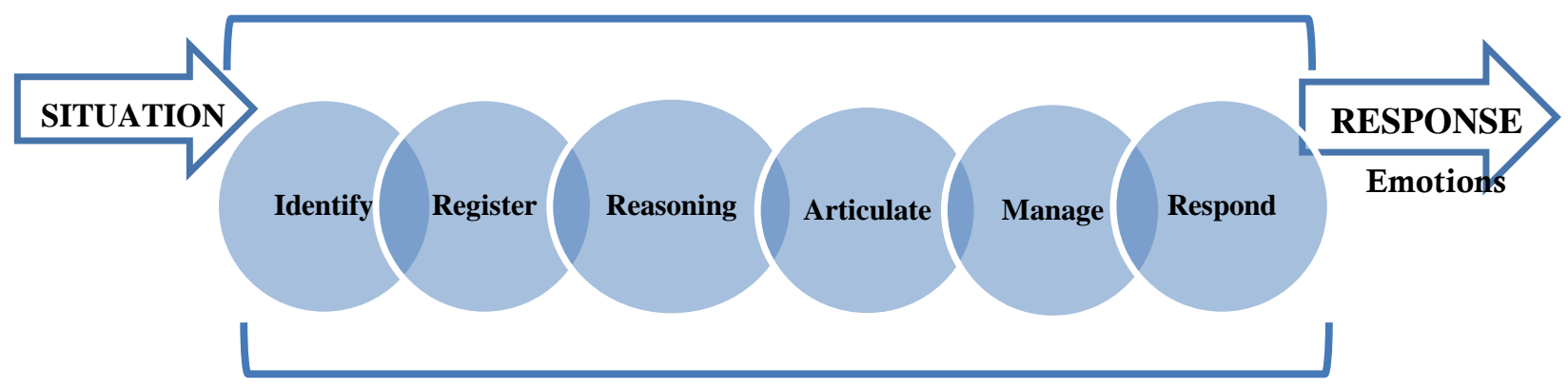

Figure 1 - Approach to enhance 'Emotional Literacy'

\section{CONCLUSIONS}

This study has aimed to develop an approach to enhance emotional literacy. In this approach, the focus is on individual's emotions. The effective use of this approach would help for individual's mental health. Emotional intelligence could be further developed by understanding others' emotions and dealing with them. Future research may wish to focus on redesigning the approach, suitable for specific stage of life. Further, intensifying constructive emotions, transforming negative emotions, developing healthy thought process, connecting with people, sharing ideas, thoughts and experiences could lead to 'best practices' in emotional management.

\section{REFERENCES AND FURTHER READING}

1. Ortony A, Turner TJ. What's basic about basic emotions?. Psychol Rev 1990;97(3):315-33.

2. Plutchik R, Kellerman H, editors. Emotion: Theory, research and experience. Vol. 1, Theories of emotion. Academic Press; 1980.

3. Erikson EH. Childhood and society. WW Norton \& Company; 1993.

4. Plutchik R. Emotion: A psychoevolutionary synthesis. Harper Collins College Division; 1980.

5. Dalgleish T, Power M, editors. Handbook of cognition and emotion. John Wiley \& Sons; 2000.

6. Reisenzein R. Arnold's theory of emotion in historical perspective. Cogn Emot 2006;20(7):920-51.

7. Shaver P, Schwartz J, Kirson D, O'connor C. Emotion knowledge: further exploration of a prototype approach. J Personal Soc Psychol 1987;52(6):1061-8.

8. Scherer KR, Schorr A, Johnstone T, editors. Appraisal processes in emotion: Theory, methods, research. Oxford University Press; 2001.

9. Lewis M, Haviland-Jones JM, Barrett LF, editors. Handbook of emotions. Guilford Press; 2010.

10. Strongman KT. The psychology of emotion. John Wiley \& Sons; 1987.

$* * * * * * * * * * * * * * * * * * * *$

Acknowledgements - Nil

Conflict of Interest $-\mathrm{Nil}$

Funding - Nil 